\title{
Short-term prognostic value of TAPSE, RVFAC and Tricuspid S' wave peak systolic velocity after first acute myocardial infarction
}

\author{
Engy Magdy Lewis Awad*, Adel Hamdy Mahmoud, Khaled Sayed Maghrby, Nasser Mohamad Taha \\ and Alaa Mohamed Ibrahim
}

\begin{abstract}
Objectives: Right ventricular dysfunction impacts the prognosis of various heart diseases. We set-out to examine which right ventricular functional parameters after STEMI and NSTEMI have prognostic value. Of 297 eligible participants, 266 (149 STEMI and 117 NSTEMI) completed follow-up. All patients underwent Grace score and 2D-echocardiography within $24 \mathrm{~h}$. Outcome was defined as occurrence of Major Adverse Cardiovascular events (MACE), such as death, recurrent ischaemia, arrhythmia, reinfarction, stroke or heart failure, within 30 days. Patients were categorized into patients with MACE and patients without MACE.

Results: In STEMI-patients, compared to those without MACE, patients with MACE experienced higher grace score, left ventricle (LV) end-systolic volume, LV end-systolic dimension and wall motion score index values, but lower tricuspid annular plane systolic excursion, right ventricle (RV) fractional area change, Tricuspid S' wave peak systolic velocity and LV ejection fraction. Nevertheless, in NSTEMI-patients, those with MACE exhibited higher left atrial volume index values, but lower tricuspid annular plane systolic excursion, RV fractional area change, $S^{\prime}$ wave peak systolic velocity and LVEF. Right ventricular fractional area change $<37.5 \%$, tricuspid annular plane systolic excursion $<15.8 \mathrm{~mm}$ and Tricuspid S' peak systolic velocity $<9.67 \mathrm{~cm} / \mathrm{s}$ are independent predictors of MACE within first 30 days after STEMI and NSTEMI.
\end{abstract}

Keywords: RVFAC, TAPSE, S' wave peak systolic velocity, AMI, NSTEMI, Short-term prognosis

\section{Introduction}

Compared to the conclusive data on the role of LV in various heart disease, the available information regarding the RV function and its role in heart diseases particularly acute myocardial infarction (AMI), is limited but outlined its significant contribution [1]. A link between right ventricular dysfunction and the prognosis of various heart disease such as heart failure and pulmonary hypertension, was reported [1]. It has been postulated that events in one ventricle affect the contralateral ventricle. Moreover, common pericardium, the in-series

*Correspondence: Engy.Magdy@mu.edu.eg

Cardiology Department, Minia University, El-Minia, Egypt circulation, interventricular septum and myocardial tracts running between the ventricles can explain the physiology of ventricular-ventricular interactions [2].

The association between right ventricular functional parameters and STEMI [3-7], but not NSTEMI, was previously examined. The association between the RV function and the outcome in patients with STEMI and NSTEMI is an issue that needs to be addressed in a large cohort of patients. Therefore, we set out to examine the RV functional parameters of acute STEMI or NSTEMI, within the first $24 \mathrm{~h}$ after the event. The relationship between those parameters and the prognosis after AMI was also investigated. 


\section{Main text}

\section{Patients and methods}

This is a prospective non-randomized study. Approval was obtained from hospital ethics committee. Patients who were admitted with AMI to our CCU from April 2014 to December 2016 were eligible to be enrolled. Consent was obtained from eligible participants. Those with co-morbidities such as renal failure, hepatic failure, corpulmonale, dilated cardiomyopathy, connective tissue diseases, pulmonary hypertension, pulmonary embolism, previous MI, permanent pacemaker or right ventricular infarction were excluded.

Of 297 patients, 266 (89.56\%) completed the follow up, of whom 117 (43.98\%) had NSTEMI and 149 (56.02\%) experienced STEMI. In patients with STEMI, 83 of 149 (55.70\%) and 66 of 149 (44.30\%) had anterior AMI and inferior AMI, respectively. All participants underwent clinical examination (HR, ABP, Killip classification) and blood tests including plasma creatinine, blood glucose, and serum hemoglobin values. Grace risk score was calculated. Echocardiography and tissue Doppler were performed, within the first $24 \mathrm{~h}$ of the event, using Vivid 3 (Vingmed, General Electric, Horton, Norway). Data acquisition was performed at a depth of $16 \mathrm{~cm}$ in the parasternal and apical views using a 3.5-MHz transducer.

The LV end-systolic volume (LV ESV) and end-diastolic volume (LV EDV) were assessed by biplane Simpson method from the apical 4- and 2-chamber views tracing the LV endocardial-blood pool interface at end-systole and end-diastole. Echocardiographic measurements were performed by expert blinded operator. LV ejection fraction (LVEF) was calculated (EF =EDV - ESV/EDV), using the biplane Simpson method. Tricuspid annular plane systolic excursion (TAPSE) was measured in the M-mode view, in the RV free wall as total displacement of the RV base from end-diastole to end-systole. RVFAC was obtained by tracing the RV end-diastolic area (RVEDA) and endsystolic area (RVESA) in the apical 4-chamber view using the formula (RVEDA - RVESA)/RVEDA $\times 100$. RV basal diameter is the maximal transverse dimension in the basal one-third of RV inflow at end-diastole in the RV-focused view. Tricuspid S' peak systolic velocity: pulsed-wave DTI at tricuspid annulus, obtained from the apical approach with parallel alignment of Doppler beam with RV free wall longitudinal excursion. LAVI was performed using the LA areas and lengths through tracing of LA endocardial borders in both the apical four- and two-chamber views. As LAV is dependent on gender, indexing to BSA was performed to abolish confounding.

Patients were followed up for 30 days after AMI event and adverse outcome was defined as occurrence any of the major adverse cardiovascular events (MACE) events during the follow up. MACE events include death, hospitalization for recurrent ischemia, reinfarction, hospitalization for heart failure, arrhythmias requiring hospital management, ischemic stroke. Patients were classified into "patients with MACE" group (patients who experienced any of the MACE events within first 30-days period after AMI) and "patients without MACE" group.

Statistical methods: The sample size was calculated with margin of error accepted at 5\% and a degree of confidence of $95 \%$. The population size was considered infinite and the response distribution was set at 25\%. All statistical calculations were performed using SPSS 22. Descriptive data was presented as mean and Standard Deviation (SD). P value $<0.05$ was considered significant. Student t-test was used to compare the mean of clinical, laboratory and doppler variables between the groups. Variable which were significant by t-test, were examined as predictors of the outcome, by using univariate regression analysis. This was followed by multivariate logistic regression analysis to determine the independent predictors of the outcome. Receiver operator characteristic analysis was used to determine the cutoff values for TAPSE, RVFAC and S' peak systolic velocity.

\section{Results}

In total 266 patients were examined, mean (SD) age 61.19 (7.97) years. Of these 266 individuals, 181 (68\%) were male, 79 (29.7\%) have diabetes mellitus, 74 (7.2\%) were smokers, 66 (24.8\%) experienced hypertension and 19 (7.1\%) exhibited positive family history of sudden cardiac death. The difference between patients with MACE and those without MACE in terms of clinical and laboratory data, Grace risk assessment score and doppler parameters is illustrated in Tables 1, 2 .

In these 266 patients, univariate regression analysis showed a significant association between MACE and LVEF, LAVI, TAPSE, RVFAC, S' wave peak systolic velocity, LV ESV and Grace score $(\mathrm{p}<0.05)$. However, by using multivariate regression, LVEF ( $p$ value $=0.032$ ), TAPSE $(\mathrm{p}=0.009)$, RVFAC $(\mathrm{p}=0.012)$ and $\mathrm{S}^{\prime}$ wave peak systolic velocity $(\mathrm{p}=0.001)$ remained significant as independent predictor for the adverse outcomes.

Of 266 patients, 149 (56.02\%) had STEMI and 117 (43.98\%) experienced NSTEMI. In those with STEMI, MACE occurred in 51 of 149 (34.22\%) patients; arrythmia required admission [7 (4.70\%)], hospitalization for recurrent ischemia [5 (3.35\%)], recurrent infarction [4 (2.68\%), hospitalization for heart failure [6 (4.03\%)], cardiogenic shock [4 (2.68\%)], stroke [2 (1.34\%)] and death [23 (15.44\%)]. However, in those with NSTEMI, MACE events were reported in 55 of 117 (47\%); arrythmia require hospital management [11 (9.4\%)], hospitalization for recurrent ischemia [7 (6\%)], reinfarction [6 $(5.1 \%)]$, hospitalization for heart failure [5 $(4.3 \%)]$, cardiogenic shock [5 (4.3\%)] and death [21 (17.9\%)]. 
Table 1 Comparison between patients with MACE and patients without MACE regarding clinical and laboratory data and Grace risk assessment score on admission

\begin{tabular}{|c|c|c|c|}
\hline & $\begin{array}{l}\text { Patients with MACE } \\
{[n=106,(39.8 \%)]}\end{array}$ & $\begin{array}{l}\text { Patients without MACE } \\
{[n=160,(60.2 \%)]}\end{array}$ & P value* \\
\hline & Mean (SD) & Mean (SD) & \\
\hline Age (years) & $61.36(6.941)$ & $61.08(8.60)$ & 0.78 \\
\hline Heart rate (Bpm) & $85.98(21.43)$ & $88.44(18.09)$ & 0.32 \\
\hline Systolic blood pressure $(\mathrm{mmHg})$ & $127.36(26.69)$ & $129.438(23.54)$ & 0.51 \\
\hline Serum creatinine (mg/dl) & $1.19(0.38)$ & $1.24(0.43)$ & 0.32 \\
\hline Random blood sugar (mg/dl) & $203.09(90.38)$ & $200.43(85.50)$ & 0.81 \\
\hline Hemoglobin (gm/dl) & $12.06(1.40)$ & $12.13(1.41)$ & 0.71 \\
\hline Grace score & $135.38(37.49)$ & $126.14(33.20)$ & $0.036^{*}$ \\
\hline
\end{tabular}

Patients with MACE: Patients who died or have experienced events in whole 30-days period post AMl; Patients without MACE: Patients who survived and have not experience events in whole 30 -days period post AMI

* $\mathrm{p}$ value $<0.05$

Table 2 Comparison between patients with MACE, patients without MACE regarding echocardiography or tissue Doppler parameters

\begin{tabular}{lccl}
\hline & $\begin{array}{l}\text { Patients } \\
\text { with MACE } \\
\text { [n=106, (39.8\%)] } \\
\text { Mean (SD) }\end{array}$ & $\begin{array}{l}\text { Patients } \\
\text { without MACE } \\
\text { [n=160, (60.2\%)] } \\
\text { Mean (SD) }\end{array}$ & (P value)* \\
\hline RVBD & $3.42(0.47)$ & $3.41(0.44)$ & 0.88 \\
RVLD & $7.82(0.23)$ & $7.82(0.23)$ & 0.89 \\
WMSI & $7.16(3.18)$ & $6.68(2.64)$ & 0.19 \\
Mitral E/A ratio & $0.98(0.34)$ & $1.02(0.35)$ & 0.49 \\
Mitral E/e' ratio & $11.32(4.23)$ & $11.28(3.18)$ & 0.92 \\
LVEF\% & $38.03(2.95)$ & $40.09(3.68)$ & $0.001^{*}$ \\
LAVI & $28.11(2.02)$ & $27.64(1.73)$ & $0.042^{*}$ \\
Tricuspid E/A ratio & $1.07(0.34)$ & $1.05(0.36)$ & 0.67 \\
Tricuspid E/e' ratio & $6.73(2.45)$ & $6.44(1.95)$ & 0.29 \\
S' wave PTSV & $10.18(1.79)$ & $11.39(2.17)$ & $0.001^{*}$ \\
(cm/s) & & & \\
TAPSE & $1.52(0.13)$ & $1.70(0.17)$ & $0.001^{*}$ \\
RVFAC\% & $36.41(2.17)$ & $39.27(2.97)$ & $0.001^{*}$ \\
LV ESd (mm) & $29.56(2.27)$ & $29.03(2.20)$ & 0.06 \\
LV EDd (mm) & $48.58(2.05)$ & $48.65(2.08)$ & 0.79 \\
LV EDV (ml) & $147.29(12.44)$ & $144.43(11.86)$ & 0.06 \\
LV ESV (ml) & $46.66(6.34)$ & $44.57(6.33)$ & $0.009^{*}$ \\
\hline Patens $w$ (m) & & &
\end{tabular}

Patients with MACE: Patients who have experienced MACE events within 30-days period after AMI. Patients without MACE: Patients who have not experience events in whole 30-days period post AMI. LVEF\%: left ventricular ejection fraction; LAVI: left atrial volume index; RVFAC: right ventricular fractional area change; LV ESd: left ventricular end-systolic diameter; LV EDd left ventricular end-diastolic diameter; LV EDV (left ventricular end-diastolic volume, LV ESV: left ventricular end-systolic volume. RVLD: RV longitudinal diameter; RVBD: Rt ventricular basal diameter; $S^{\prime}$ wave PTSV: $S^{\prime}$ wave peak tricuspid systolic velocity; TAPSE: tricuspid annular plane systolic excursion; WMSI: wall motion score index * $p$ value $<0.05$
Table 3 showed that in patient with STEMI, TAPSE, RVFAC, S' wave peak systolic velocity, LVEF values, LV ESV, LV ESd, WM score index and Grace score were significantly different between individuals with MACE and those without MACE. However, in those with NSTEMI, only LAVI, TAPSE, RVFAC, S' wave peak systolic velocity and LVEF values were significantly different between patients with and without MACE.

The link between MACE events and echocardiography parameters and doppler values was examined. In patients with STEMI, by using univariate regression, MACE was significantly associated with Grace score, TAPSE, S' wave peak systolic velocity, RVFAC, LVEF, LV ESV, LV ESd and WMSI (Wall Motion Score Index) ( $\mathrm{p}$ value $<0.05$ ). However, by using multivariate regression analysis, only TAPSE, S' wave peak systolic velocity and RVFAC remained significant ( $p$ value $<0.05$ ). Similarly, in those with NSTEMI, using univariate regression analysis showed that there was a significant association between the adverse outcome and LVEF, TAPSE, RVFAC, S' wave peak systolic velocity and LAVI ( $p$ value $<0.05$ ), notably this association was not documented with Grace score, LV ESV, LVESd or WMSI ( $\mathrm{p}$ value $>0.05$ ). However, by using multivariate regression analysis, LVEF ( $\mathrm{p}$ value $0.041)$, TAPSE $(p=0.001), \operatorname{RVFAC}(p=0.001)$ and $S^{\prime}$ wave peak systolic velocity $(p=0.008)$ remained significant as independent predictor for the adverse outcomes.

By the end of the first 30 days post AMI, the overall MACE events were noticed in $64.8 \%$ of patients who have RV dysfunction $(\mathrm{n}=54)$ (defined as $\mathrm{RVFAC}<35 \%)$, but 
Table 3 Mean (SD) of Grace score, echocardiography parameters and tissue doppler values between the two groups of patients (patients with MACE and patients without MACE) in patients with STEMI and NSTEMI

\begin{tabular}{|c|c|c|c|c|c|c|}
\hline & STEMI $(n=149)$ & & & NSTEMI $(n=117)$ & & \\
\hline & $\begin{array}{l}\text { Patients with MACE } \\
\text { [mean (SD)] }\end{array}$ & $\begin{array}{l}\text { Patients without MACE } \\
\text { [mean (SD)] }\end{array}$ & P value ${ }^{\S}$ & $\begin{array}{l}\text { Patients with MACE } \\
\text { [mean (SD)] }\end{array}$ & $\begin{array}{l}\text { Patients without MACE } \\
\text { [mean (SD)] }\end{array}$ & Pvalue ${ }^{\S}$ \\
\hline Grace score & $140.27(41.46)$ & $123.81(34.10)$ & $0.01^{\S}$ & 130.84 (33.13) & $129.82(31.65)$ & 0.87 \\
\hline Mitral E/A ratio & $0.97(0.32)$ & $0.99(0.36)$ & 0.72 & $0.99(0.37)$ & $1.06(0.33)$ & 0.41 \\
\hline Mitral E/e' ratio & $11.23(4.35)$ & $11.37(3.24)$ & 0.85 & $11.40(4.15)$ & $11.16(3.10)$ & 0.72 \\
\hline Tricuspid E/A & $1.08(0.33)$ & $1.03(0.34)$ & 0.38 & $1.06(0.33)$ & $1.08(0.38)$ & 0.69 \\
\hline Tricuspid E/e' & $6.96(2.94)$ & $6.55(1.91)$ & 0.30 & $6.51(1.87)$ & $6.27(2.01)$ & 0.50 \\
\hline RVBD & $3.41(0.50)$ & $3.44(0.43)$ & 0.64 & $3.42(0.43)$ & $3.35(0.46)$ & 0.37 \\
\hline RVLD & $7.76(0.21)$ & $7.81(0.24)$ & 0.21 & $7.88(0.23)$ & $7.89(0.23)$ & 0.51 \\
\hline WMSI & $8.61(2.85)$ & $7.30(2.53)$ & $0.005^{\S}$ & $5.81(2.88)$ & $5.71(2.51)$ & 0.84 \\
\hline TAPSE (cm) & $1.50(0.12)$ & $1.68(0.16)$ & $0.001^{\S}$ & $1.53(0.14)$ & $1.72(0.18)$ & $0.001^{\S}$ \\
\hline S' wave PTSV $(\mathrm{cm} / \mathrm{s})$ & $9.80(1.21)$ & $11.12(2.04)$ & $0.002^{\S}$ & $10.52(2.15)$ & $11.81(2.32)$ & $0.003^{\S}$ \\
\hline RVFAC (\%) & $36.48(2.15)$ & $39.03(2.91)$ & $0.001^{\S}$ & $36.35(2.21)$ & $39.65(3.06)$ & $0.005^{\S}$ \\
\hline LV EF (\%) & $38.18(3.04)$ & $40.23(3.77)$ & $0.02^{\S}$ & $37.90(2.88)$ & $39.80(3.55)$ & $0.002^{5}$ \\
\hline LAVI & $28.25(2.20)$ & $27.85(1.66)$ & 0.21 & $27.99(1.87)$ & $27.31(1.80)$ & $0.041^{\S}$ \\
\hline LV ESd (mm) & $29.92(2.05)$ & $28.97(2.24)$ & $0.013^{\S}$ & $29.23(2.43)$ & $29.14(2.14)$ & 0.82 \\
\hline LV EDd (mm) & $48.91(2.04)$ & $48.67(2.07)$ & 0.501 & $48.28(2.03)$ & $48.62(2.13)$ & 0.37 \\
\hline LV EDV (ml) & $147.22(11.59)$ & $143.24(11.76)$ & 0.051 & $147.36(13.2)$ & $146.32(11.87)$ & 0.66 \\
\hline LVESV (ml) & $46.58(5.64)$ & $44.51(6.38)$ & $0.045^{\S}$ & $46.73(6.98)$ & $44.66(6.30)$ & 0.09 \\
\hline
\end{tabular}

LAVI: left atrial volume index; LV EF: left ventricular ejection fraction; RVBD: Rt ventricular basal diameter; RVFAC: Right ventricular fractional area change; RVLD: RV longitudinal diameter; S' wave PTSV: S' peak tricuspid systolic velocity; TAPSE: Tricuspid annular plane systolic excursion; WMSI: wall motion score index

$\S$ p value $<0.05$

only in $33.5 \%$ of patients with normal RV function $(n=212)$ post AMI [odds ratio 3.65 (95\% CI 1.95-6.85), $\mathrm{P}<0.001$ ].

Receiver operator characteristic (ROC) analysis was used to examine the cutoff of various variables and their sensitivity and specificity in predicting the adverse outcome. We have noted that the TAPSE cutoff value was $15.8 \mathrm{~mm}$, with area under the curve (AUC) $0.803,95 \% \mathrm{CI}$ (0.749-0.859), sensitivity of $79.3 \%$ and specificity $75.1 \%$. The cutoff value for RVFAC was $37.5 \%$, with AUC 0.783), 95\% CI (0.727-0.839), sensitivity $72.6 \%$ and specificity $73.7 \%$. S' peak systolic velocity cutoff value was $9.67 \mathrm{~cm} / \mathrm{s}$, AUC 0.783, 95\% CI (0.670-0.797), 73\% sensitivity and 65.0\% specificity), (Additional file 1: Fig S1).

\section{Discussion}

In the current study, we have demonstrated the right ventricular functional parameters within the first $24 \mathrm{~h}$ from AMI and their relation to MACE in those presented with STEMI or NSTEMI. In STEMI and NSTEMI patients; a link has been observed between RV dysfunctional parameters such as RVFAC, TAPSE and S' peak systolic velocity and occurrence of MACE events within the first 30 days after AMI. In patients with STEMI and NSTEMI, individuals with MACE had lower TAPSE, RVFAC, S' wave peak systolic velocity and LVEF values than those without MACE. However, they experienced higher Grace score, LVESd, LVESV and WMSI values, in patients with STEMI, and higher LAVI values in those with NSTEMI.

Emerging evidence suggests that the right ventricular dysfunction is an important predictor of cardiac events and mortality in multiple cardiac diseases $[1,8]$. In this cohort of patients, RV dysfunction parameters such as TAPSE, RVFAC, and S' wave peak systolic velocity of the tricuspid valve were independent predictors for MACE outcome within first 30 days after acute AMI.

In this cohort of patients, we have found that right ventricular dysfunction occurs in individuals with inferior as well as anterior infarction, in accordance with previous studies $[3,4,9]$. It was suggested that LAD occlusion results in right ventricular dysfunction through impaired septal contractility as a result of decreased supply through septal perforators and ischemia of posterior aspect of apical septum [3]. Moreover, impaired RV function could occur through increased afterload after LV dysfunction from anterior infarction [3]. In patients studied with Cardiac Magnetic Resonance, right ventricular ischemic changes were found adjacent to the ischemic dysfunctional left ventricular myocardium [5]. Moreover, this pattern was observed in inferior left ventricular infarcts as well as in up to $33 \%$ of anterior left ventricular 
infarcts, with an impact on right ventricular performance [5].

We noted a link between MACE event and RV dysfunctional parameters such as RVFAC and TAPSE. Similarly, the association between RVFAC or TAPSE and adverse outcome has been reported in previous study, in patients with AMI [7]. In patients with acute inferior STEMI, an association between TAPSE value $\leq 14 \mathrm{~mm}$ and adverse outcome was demonstrated in previous study [10]. However, Lohitashwa and colleagues have been shown that RVFAC, but not TAPSE, was associated with adverse outcome in setting of STEMI [6]. Moreover, in STEMI patients, particularly those with impaired left ventricular function in the context of anterior MI, a link between right ventricular dysfunction and ventricular tachycardia has been suggested [3]. Furthermore, the severity of right ventricular dysfunction after AMI has significant impact on long-term mortality [11].

In this cohort of patients, TAPSE and RVFAC are independent predictors for MACE after STEMI, in accordance with the previous studies $[4,10]$. Interestingly, we have noted that TAPSE and RVFAC are independent predictors for MACE after NSTEMI as well. In addition, Tricuspid S' wave peak systolic velocity was independent predictor of MACE within first 30 days after both STEMI and NSTEMI.

\section{Conclusion}

Compared to patients without MACE, patients with MACE had lower TAPSE, RVFAC, S' wave peak systolic velocity and LVEF values, in patients with STEMI and NSTEMI. However, they experienced higher Grace score, LVESV, LVESd and WMSI values in patients with STEMI, and higher LAVI values in those with NSTEMI.

In STEMI and NSTEMI patients; RV dysfunctional parameters in terms of RVFAC $<37.5 \%$, TAPSE $<15.8 \mathrm{~mm}$ and S' peak systolic velocity $<9.67 \mathrm{~cm} / \mathrm{s}$ are independent predictors of MACE events within the first 30 days after AMI. These parameters should be used in clinical settings to identify those with high risk.

\section{Limitation}

Single center-based study.

Data regarding the invasive catheterization was not available for all patients.

\section{Supplementary information}

Supplementary information accompanies this paper at https://doi. org/10.1186/s13104-020-05040-2.

Additional file 1: Fig. S1. ROC curve for TAPSE, RVFAC, S' peak systolic velocity.

\section{Abbreviations}

AMI: Acute myocardial infarction; DTI: Doppler tissue imaging; MACE: Major adverse cardiovascular events; LAD: Left anterior descending; LA: Left atrium; LAVI: Left atrial volume index; LV: Left ventricle; LV EDd: Left ventricular enddiastolic diameter; LV EDV: Left ventricular end-diastolic volume; LVEF: Left ventricular ejection fraction; LV ESd: Left ventricular end-systolic diameter; LV ESV: Left ventricular end-systolic volume; NSTEMI: Non-ST segment elevation myocardial infarction; RCA: Right coronary artery; RV: Right ventricle; RVBD: Rt ventricular basal diameter; RVEDA: Right ventricular end diastolic area; RVESA: Right ventricular end-systolic area; RVFAC: Right ventricular fractional area change; RVLD: RV longitudinal diameter; S' wave PTSV: S' wave peak tricuspid systolic velocity; SD: Standard deviation; STEMI: ST segment elevation myocardial infarction; TAPSE: Tricuspid annular plane systolic excursion; WMSI: Wall motion score index.

\section{Acknowledgements}

No Acknowledgment related this study.

\section{Authors' contributions}

EMLA: involved in collecting the data, writing the manuscript and performing the statistical analysis. AHM: Revised and edited the manuscript, involved in the statistical analysis and supervised the study. KSM: Revised and edited the manuscript, involved in the statistical analysis and supervised the study. NMT: Revised and edited the manuscript, involved in the statistical analysis and supervised the study. AMI: Revised and edited the manuscript, involved in the statistical analysis and supervised the study. All authors read and approved the final manuscript.

Funding

No funding was received by any of the authors of this research work

\section{Availability of data and materials}

The datasets used and/or analyzed during the current study are available from the corresponding author on reasonable request.

\section{Ethics approval and consent to participate}

Approval was obtained from El-Minia University hospital ethics committee, Egypt.Written informed consent has been obtained from participants.

\section{Consent to publish}

Not applicable.

\section{Competing interests}

No competing interest to declare.

Received: 10 February 2020 Accepted: 21 March 2020

Published online: 01 April 2020

\section{References}

1. Voelkel NF, Quaife RA, Leinwand LA, Barst RJ, McGoon MD, Meldrum DR, National Heart, Lung, and Blood Institute Working Group on Cellular and Molecular Mechanisms of Right Heart Failure, et al. Right ventricular function and failure: report of a National Heart, Lung, and Blood Institute working group on cellular and molecular mechanisms of right heart failure. Circulation. 2006;114:1883-91. https://doi.org/10.1161/circulatio naha.106.632208.

2. Friedberg MK. Imaging right-left ventricular interactions. JACC CardiovasC Imaging. 2018;11(5):755-71. https://doi.org/10.1016/j.jcmg.2018.01.028.

3. Thakkar JB, Zaman S, Byth K, Narayan A, Thiagalingam A, Chow C, et al. Right ventricular dysfunction predisposes to inducible ventricular tachycardia at electrophysiology studies in patients with acute stsegment-elevation myocardial infarction and reduced left ventricular ejection fraction. Circ Arrhythm Electrophysiol. 2014;7:898-905. https:// doi.org/10.1161/CIRCEP.113.001594.

4. Gorter TM, Lexis CP, Hummel YM, Lipsic E, Nijveldt R, Willems TP, et al. Right ventricular function after acute myocardial infarction treated with primary percutaneous coronary intervention (from the glycometabolic intervention as adjunct to primary percutaneous coronary intervention 
in ST-segment elevation myocardial infarction III trial. Am J Cardiol. 2016;118:338-44.

5. Masci PG, Francone M, Desmet W, Ganame J, Todiere G, Donato R, et al. Right ventricular ischemic injury in patients with acute ST-segment elevation myocardial infarction characterization with cardiovascular magnetic resonance. Circulation. 2010;122:1405-12. https://doi.org/10.1161/CIRCU LATIONAHA. 110.940254.

6. Lohitashwa SB, Srilakshmi MA, Varghese K, Santhosh MJ, Raghavan SR Echocardiographic assessment of right ventricular function in acute myocardial infarction. Indian Heart J. 2014;66:1-43.

7. Antoni ML, Scherptong R, Atary JZ, Boersma E, Holman ER, van der Wall $\mathrm{EE}$, et al. Prognostic value of right ventricular function in patients after acute myocardial infarction treated with primary percutaneous coronary intervention. Circ Cardiovasc Imaging. 2010;3:264-71. https://doi. org/10.1161/CIRCIMAGING.109.914366.

8. Aschauer S, Kammerlander AA, Zotter-Tufaro C, Ristl R, Pfaffenberger S, Bachmann A, et al. The right heart in heart failure with preserved ejection fraction: insights from cardiac magnetic resonance imaging and invasive hemodynamics. Eur J Heart Fail. 2016;18(1):71-80. https://doi. org/10.1002/Ejhf.418.
9. Jensen CJ, Jochims M, Hunold P, Sabin GV, Schlosser T, Bruder O. Right ventricular involvement in acute left ventricular myocardial infarction: prognostic implications of MRI findings. AJR Am J Roentgenol. 2010;194:592-8. https://doi.org/10.2214/AJR.09.2829.

10. Hayrapetyan HG, Yerevan MC. Tricuspid annular plane systolic excursion in acute left ventricular inferior myocardial infarction with ST segment elevation: prognostic importance and influence on ergometric parameters. Eur Heart J. 2011;32:1063.

11. Shahar K, Darawsha W, Yalonetsky S, Lessick J, Kapeliovich M, Dragu R, et al. Time dependence of the effect of right ventricular dysfunction on clinical outcomes after myocardial infarction: role of pulmonary hypertension. J Am Heart Assoc. 2016;5(7):e003606. https://doi.org/10.1161/ jaha.116.003606.

\section{Publisher's Note}

Springer Nature remains neutral with regard to jurisdictional claims in published maps and institutional affiliations.
Ready to submit your research? Choose BMC and benefit from:

- fast, convenient online submission

- thorough peer review by experienced researchers in your field

- rapid publication on acceptance

- support for research data, including large and complex data types

- gold Open Access which fosters wider collaboration and increased citations

- maximum visibility for your research: over 100M website views per year

At BMC, research is always in progress.

Learn more biomedcentral.com/submissions 